\title{
Computational Analysis of High-Altitude Ionization Gauge Flight Measurements
}

\author{
Quanhua Sun, ${ }^{*}$ Chunpei Cai, ${ }^{\dagger}$ and Iain D. Boyd ${ }^{\ddagger}$ \\ University of Michigan, Ann Arbor, Michigan 48109 \\ and \\ James H. Clemmons ${ }^{\S}$ and James H. Hecht** \\ The Aerospace Corporation, El Segundo, California 90245
}

\begin{abstract}
The rarefied, three-dimensional flows experienced during the turbulent oxygen mixing experiment (TOMEX) at altitudes between 85 and $143 \mathrm{~km}$ are simulated using the direct simulation Monte Carlo (DSMC) method. The present study focuses on ionization gauge measurements obtained by TOMEX. The payload is, thus, modeled in detail, and the simulations employ complex meshes. The simulations show that a bow shock wave is generated in front of the payload at low altitude that becomes diffusive at higher altitudes. When the altitude increases, the pressure in the channels of the ionization gauge and the pressure variation around the payload are both decreased. The DSMC results agree very well with data predicted by compressible flow theory and free molecular theory when applicable. Comparison between the DSMC results and the TOMEX flight data shows generally good agreement.
\end{abstract}

\section{Nomenclature}

$H \quad=$ altitude

$K n=$ Knudsen number

$k=$ Boltzmann constant

$M \quad=$ Mach number

$m \quad=$ mass of molecules

$n \quad=$ number density

$p \quad=$ pressure

$s \quad=$ normalized velocity

$T=$ temperature

$U, V, W=$ velocity in $x, y$, or $z$ direction

$x, y, z=$ physical coordinates

$\gamma=$ ratio of specific heats

Subscripts and Superscripts

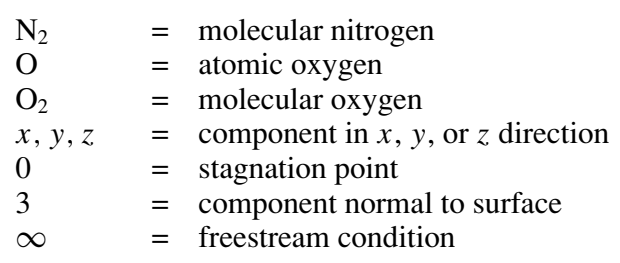

\section{Introduction}

A NUMBER of chemical and dynamic processes that have minor significance throughout much of the atmosphere play a major role in determining the structure and energetics of the atmosphere at altitudes between 80 and $110 \mathrm{~km}$, often referred to as the meso-

Presented as Paper 2004-2686 at the AIAA 37th Thermophysics Conference, Portland, OR, 28 June-1 July 2004; received 29 August 2004; revision received 16 December 2004; accepted for publication 20 December 2004. Copyright (C) 2005 by the American Institute of Aeronautics and Astronautics, Inc. All rights reserved. Copies of this paper may be made for personal or internal use, on condition that the copier pay the $\$ 10.00$ per-copy fee to the Copyright Clearance Center, Inc., 222 Rosewood Drive, Danvers, MA 01923; include the code 0022-4650/06 \$10.00 in correspondence with the CCC.

*Postdoctoral Research Fellow, Department of Aerospace Engineering. Senior Member AIAA.

${ }^{\dagger}$ Graduate Student, Department of Aerospace Engineering. Member AIAA.

${ }_{\ddagger}^{\ddagger}$ Professor, Department of Aerospace Engineering. Associate Fellow AIAA.

${ }^{\S}$ Research Scientist, Space Science Applications Laboratory.

** Senior Scientist, Space Science Applications Laboratory. sphere and lower thermosphere (MALT) region. Such processes include the production and diffusion of atomic oxygen, interactions of waves and tides, and breakdown of atmospheric gravity waves. Whereas the relationships among eddy diffusion, turbulence, instability structures, dynamic activity, and constituent transport have often been discussed and speculated on, an important question is how the species present in the MALT region are actually affected when potentially unstable conditions occur. The turbulent oxygen mixing experiment (TOMEX) ${ }^{1}$ was, therefore, designed to measure the actual atmospheric response to the existence of unstable layers as determined by wind and temperature measurements from 80 to $105 \mathrm{~km}$.

The TOMEX combined Na lidar measurements from Starfire Optical Range in Albuquerque, New Mexico, with a launch of a mother-daughter payload from White Sands Missile Range, located a little over $100 \mathrm{~km}$ from Starfire. The payload included a trimethyl aluminum release (on the mother payload) to measure winds and diffusion, a three-channel photometer experiment (on the daughter payload) to measure atomic oxygen-related airglow, and a five-channel ionization gauge (on the daughter payload) to measure neutral density fluctuations at high vertical resolution. The payload was launched at 0957 Universal Mean Time (UMT) on 26 October 2000 and successfully obtained data from all of the experiments. For instance, the ionization gauge recorded the density at five locations on the daughter payload. This kind of multilocation density measurement could infer the flow density, temperature, and velocity, in principle. The complexity of the flow, however, can make such interpretation difficult. For example, there were momentum and energy transfer on the payload surface and a bow shock wave formed in front of the payload. Furthermore, the mean free path of the atmosphere in the MALT region was comparable to the payload size, which indicates that the flow was in the transitional or even free-molecular regime. Clearly, no simple theory can account for the complicated flow experienced during the TOMEX. Numerical simulations based on kinetic theory are, therefore, necessary to interpret the measurement data because continuum-based simulations fail to predict rarefied flows.

The most popular approach for simulating rarefied gas flows is the direct simulation Monte Carlo (DSMC) method (Ref. 2) that has been widely used in the rarefied atmospheric gasdynamics as well as other fields involving low gas density or small physical length scale. The DSMC method has been successfully applied to study the external flows of many vehicles, including the Space Shuttle Orbiter, ${ }^{3}$ the Magellan Spacecraft, ${ }^{4}$ the Stardust Sample Return Capsule, ${ }^{5}$ a reentry capsule, ${ }^{6}$ the Mars Pathfinder, ${ }^{7}$ the Columbia Shuttle Orbiter, ${ }^{8}$ and the Mars Reconnaissance Orbiter. ${ }^{9}$ The DSMC method has also 
been used to analyze pressure sensor measurements by coupling external and internal flow simulations. ${ }^{10}$ However, integral simulation of both external and internal flows for a pressure sensor on a vehicle has not been reported in previous work.

The objective of this paper is to help understand both the external and internal flows experienced during the TOMEX using numerical simulations. Additional findings from the TOMEX have been reported in several other papers. ${ }^{11-14}$ In this paper, the payload geometry is first modeled (Sec. II) by including details of important structures. Next, flows resulting from the payload flight are simulated (Sec. III) by employing the DSMC method. Simulated results are then analyzed and compared with the measurement data (Sec. IV). The paper ends with some concluding remarks (Sec. V).

\section{Payload Modeling}

The rocket (mother-daughter payload) was launched from the ground when the lidar data indicated the presence of convectively and dynamically unstable regions in the atmosphere between 80 - and $100-\mathrm{km}$ altitude. After the payload reached an altitude above $65 \mathrm{~km}$, the daughter payload was separated from the mother payload at a separation speed of a few meters per second. The daughter payload traveled at about $1 \mathrm{~km} / \mathrm{s}$ through the $80-110-\mathrm{km}$ region with a spin rate of $1 \mathrm{~Hz}$. The ionization gauge sensor units installed on the daughter payload were evacuated and sealed before launch and opened by breaking the seal once the payload attained altitude. Each sensor unit of the ionization gauge consisted of a miniature sensor and an accommodation chamber, where the sensor monitored the density of the gas in the accommodation chamber. The measurement technique is based on a well-established principle: a volume of gas to be measured is partially ionized by a stream of electrons, then the resulting ions are collected by a biased collector. The gas density can be determined because it is proportional to the ratio of the emitted electron current to the collected ion current. Instruments operating on this principle have been flown in space for decades, primarily to measure the temperature of the upper atmosphere. ${ }^{15}$

To interpret the measurement data obtained by the ionization gauge, the daughter payload (called payload hereafter) and the ionization gauge must be modeled and included in numerical simulations. The payload has a cylindrical surface with several instruments installed inside and outside, as shown in Fig. 1. On the top of the

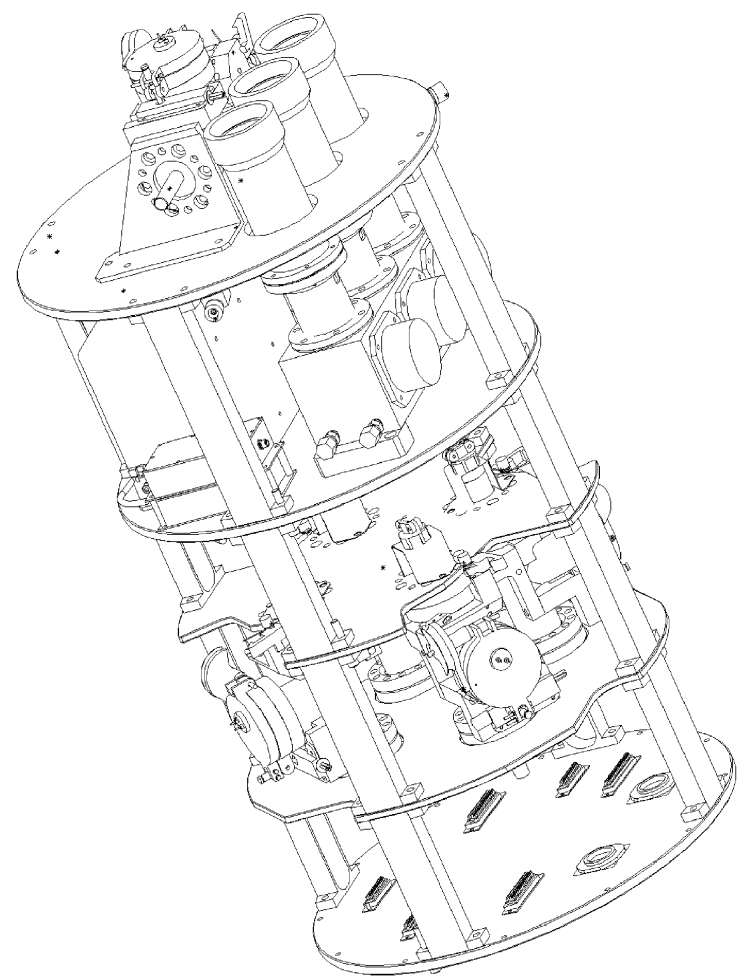

Fig. 1 The Aerospace Corporation three-channel photometer and ionization gauge daughter payload.

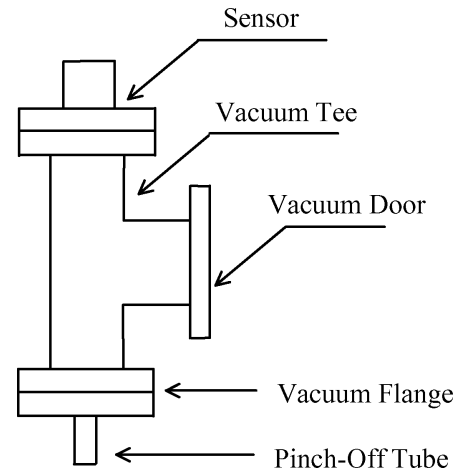

Fig. 2 Schematic of channel of ionization gauge.

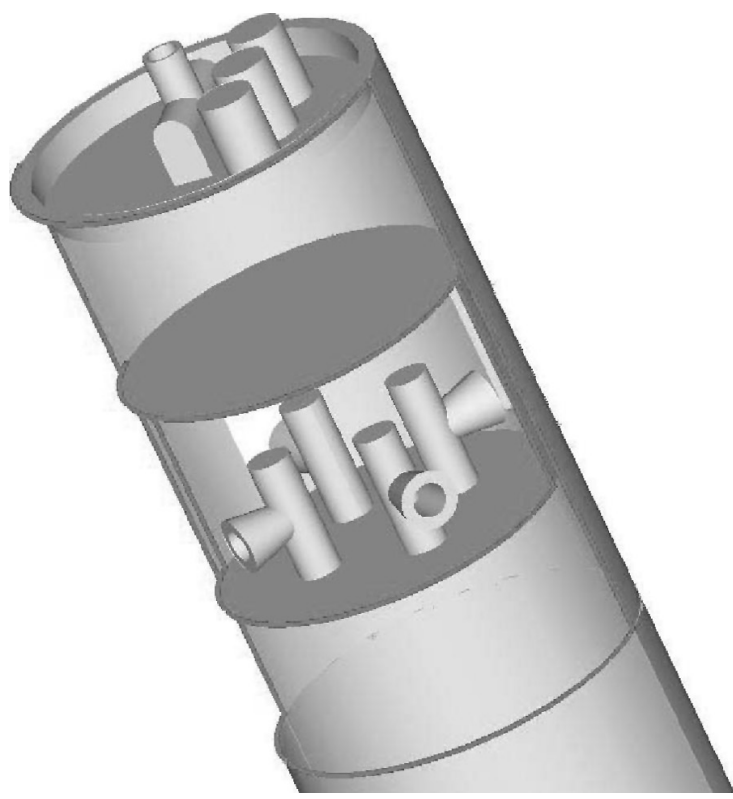

Fig. 3 Modeled daughter payload.

payload are three photometer telescopes and a forward channel of the ionization gauge. There are four equally spaced openings on the side surface of the payload where each opening faces one side channel of the ionization gauge. Three deck plates are used to mount the internal instruments. Flow is negligible through the upper and lower deck plates, whereas the cutouts in the middle deck plate take up about one-half of the deck plate area. The regions between the upper and lower deck plates are, therefore, the only internal part of the payload having significant flow from the atmosphere. The sensors have several components as shown in the schematic in Fig. 2, where the vacuum door is opened during the measurement.

The payload is modeled as shown in Fig. 3, in which part of the surface is removed to show the internal structure of the payload. Several simplifications are made by omitting unimportant details of the geometry. First, the photometer telescopes are modeled as three solid cylinders. Second, each channel of the ionization gauge is simplified, and the details are shown in Fig. 4, where the vacuum door is not included in the simulations. The sizes of the channels are as follows: The diameter of the opening is slightly larger than $0.04 \mathrm{~m}$. The vacuum tee has a diameter of $0.035 \mathrm{~m}$ and a height of $0.122 \mathrm{~m}$. The distance between the opening and the vacuum tee is about $0.058 \mathrm{~m}$. Third, the upper and lower deck plates are both treated as solid plates that separate the internal flows above the upper plate and below the lower plate from the atmosphere, and the middle deck plate is removed for simplicity. Finally, the modeled payload includes a simplified representation of the support structures located below the experiment section of Fig. 1, and the modeled payload has a total height close to $1.38 \mathrm{~m}$ with a diameter of about $0.44 \mathrm{~m}$. Nevertheless, the additional $0.63-\mathrm{m}$ payload was not expected to affect the operation of the ionization gauges because the flight of the payload was supersonic. 


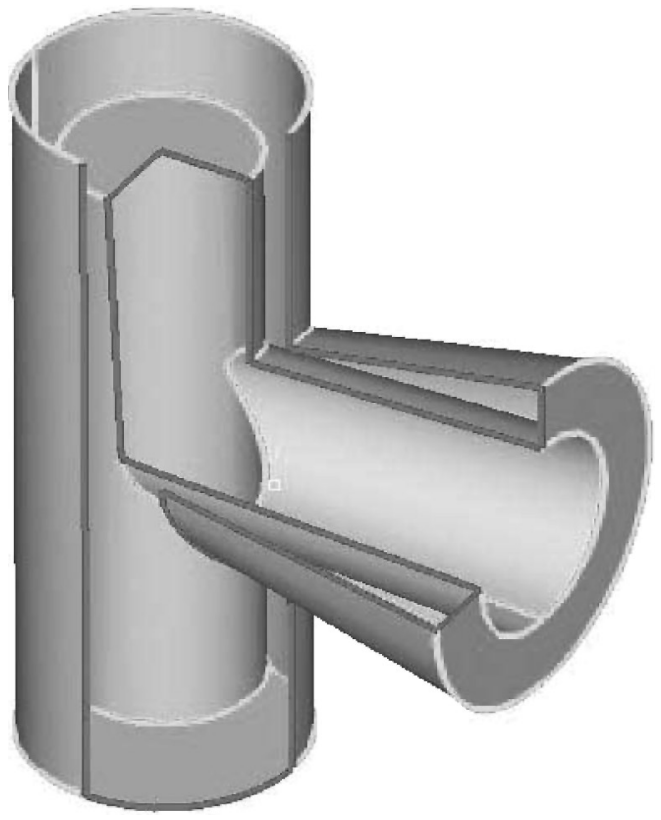

Fig. 4 Modeled ionization gauge channel.

\section{DSMC Simulation}

The TOMEX experiment was performed in the MALT region. The atmosphere in this region is very rarefied. For instance, the widely used mass-spectrometer-incoherent-scatter (MSIS-90) model ${ }^{16}$ contains the following values of mean free path: $0.01 \mathrm{~m}$ at $86 \mathrm{~km}, 0.1 \mathrm{~m}$ at $100 \mathrm{~km}, 1 \mathrm{~m}$ at $112 \mathrm{~km}$, and $10 \mathrm{~m}$ at $131 \mathrm{~km}$. All of these values are comparable to the diameter of the payload, $0.44 \mathrm{~m}$. Therefore, the DSMC method is used to simulate all of the flows.

\section{A. DSMC Method}

The DSMC method ${ }^{2}$ is a widely used particle method and is an effective numerical technique to simulate rarefied, nonequilibrium gas flows. It simulates rarefied gas flows based on physical principles instead of mathematical formulation. The DSMC method models gas flows through computations of motions and collisions of representative particles. The primary approximation is to uncouple the molecular motions and intermolecular collisions over small time intervals, in which particle motions are modeled deterministically and the collisions are treated statistically.

The flow is simulated using the state-of-the-art DSMC research code MONACO ${ }^{17}$ It is a general-purpose code for modeling twodimensional, axisymmetric, or three-dimensional rarefied gas flows. The code is fully parallelized, and the computational domain can be automatically decomposed using the partitioning package called Metis. ${ }^{18}$ The variable hard-sphere molecular model ${ }^{2}$ is specified in this case, which assumes that the total collision cross section depends on the relative collision velocity.

\section{B. Computational Mesh}

The physics of a problem determines the required computational domain and its boundary conditions, which in this case indicates that the size of the necessary computational domain should vary with altitude for the TOMEX flows. In DSMC simulations, the computational domain is divided into cells where the local cell size should be less than the local mean free path of the flow. Enough cells are also required to represent faithfully the modeled payload. Besides the physical requirements, a practical constraint is to limit the total number of computational cells due to the limitation of the computer resources. For the present flows, small cells are used around the payload, especially near the ionization gauges, and relatively large cells are employed for the rest of the computational domain where the mean free path of molecules is relatively large.

Following the foregoing principles, several tetrahedral meshes are generated using the commercial software Hypermesh. ${ }^{19}$ A typical

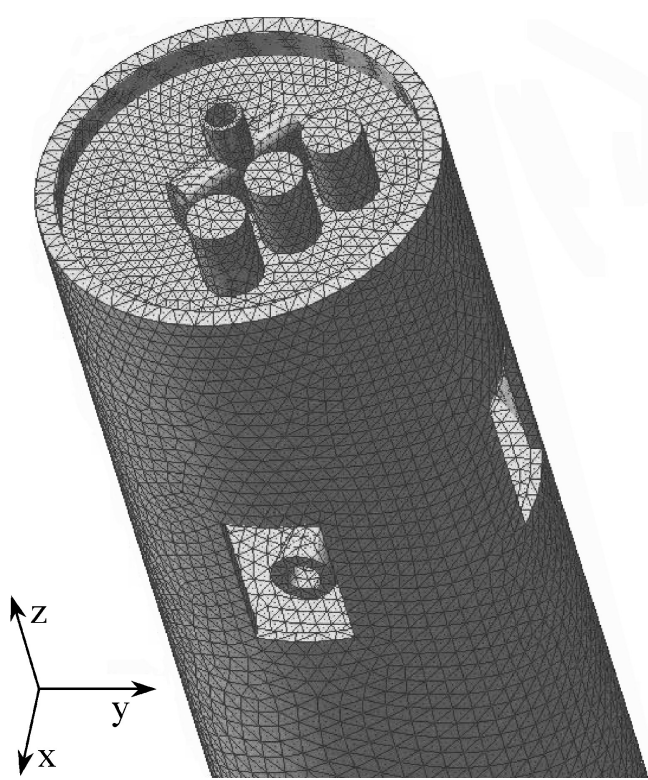

Fig. 5 Surface mesh of payload for $90-\mathrm{km}$ case.

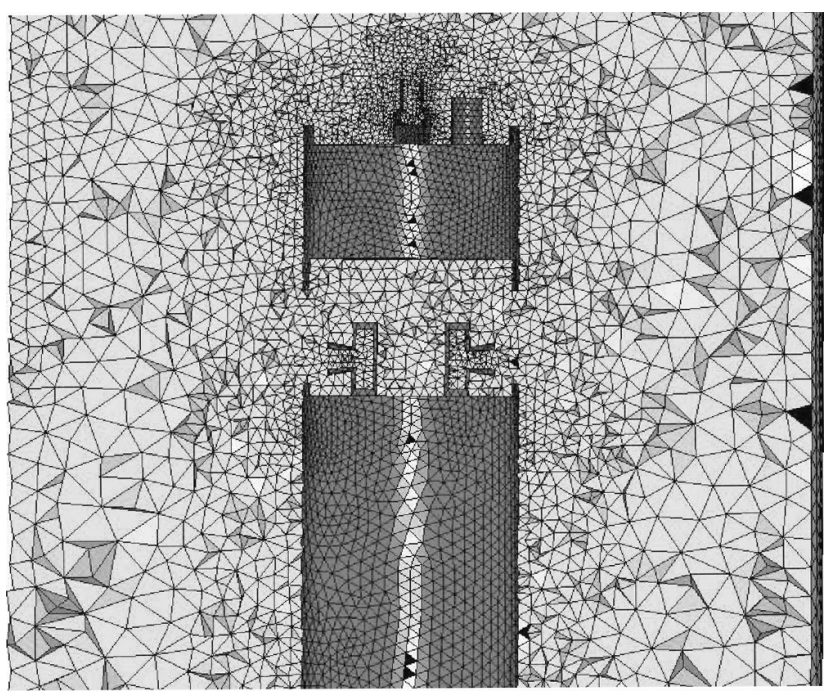

Fig. 6 Cut-open view of tetrahedral mesh for 90-km case.

surface mesh is shown in Fig. 5, in which the axis is assigned to the $z$ direction and the openings face the $x$ or $y$ direction. The surface is modeled as a solid wall. Its temperature is assumed to be $300 \mathrm{~K}$ because the sensors remained around $300 \mathrm{~K}$ because of the high heat capacity of aluminum and because the large heat conductivity of the surface kept the payload at one temperature. The thermal accommodation coefficient is set to one, which is a good approximation for the bare aluminum used. The entire surface is divided into many wall faces for the simulations: 51,750 faces for the flow at $85 \mathrm{~km}$ and 8134 faces for the flow at $120 \mathrm{~km}$. The volume of the entire computational domain is about $3.4 \mathrm{~m}^{3}$ for the $85-\mathrm{km}$ case and is increased to $405 \mathrm{~m}^{3}$ for the $120-\mathrm{km}$ case. Figure 6 shows a cut-open view of the mesh for the $90-\mathrm{km}$ case that displays the structure of the tetrahedral mesh. The total number of tetrahedral cells varies from $1,096,235$ for the $85-\mathrm{km}$ case, to 141,642 for the $100-\mathrm{km}$ case, to 572,488 for the $120-\mathrm{km}$ case.

The cell size for lower-altitude simulations is not always less than the mean free path of the atmosphere, although many cells are employed. To ensure that collisions only occur between particles within a mean free path, a near-partner selection technique is proposed and used to determine the collision particle pairs. This technique is slightly different from the subcell technique in which a cell is divided into several subcells. The subcell technique is not used 
because it is difficult to divide a three-dimensional unstructured cell and to group particles for subcells. The near-partner technique allows a particle to collide with particles only within a small distance. In the present investigation, the distance is predetermined and is less than one-half of the cell edge, which ensures that the distance is less than the local mean free path throughout the computational domain.

The payload flew at a supersonic speed below $130 \mathrm{~km}$ (speed of sound being calculated using the MSIS temperature, which should be close to those during flight), which allows simulations to employ a relatively small computational domain. However, a relatively large computational domain is required to implement effective boundary conditions for the subsonic flows experienced at higher altitude. A larger or finer grid mesh is actually used to test the grid independency of solutions to ensure a reasonable mesh is used for each simulation case.

\section{Particle Weighting}

In a DSMC simulation, the number of simulated particles is determined by the size of the computational domain and the number of real molecules that each simulated particle represents. For instance, the computational domain for the flow at $100 \mathrm{~km}$ has a volume of $12 \mathrm{~m}^{3}$, and the volume of the minimum cell (tetrahedral) is about $1 \times 10^{-7} \mathrm{~m}^{3}$. If 20 particles are simulated in the minimum cell, a total of $2.4 \times 10^{9}$ particles have to be simulated at the same time if the density is assumed constant throughout the domain. Such a simulation is extremely expensive based on the available computational resources. To limit the total number of simulated particles, a particle weighting strategy is employed. In general, a particle in larger cells represents more real molecules, such that a similar number of particles are simulated in each cell. This is achieved by assigning a relative particle weight to each cell.

Because the computational cells have different particle weights, a particle may be destroyed or cloned when it moves into another cell. The destroying and cloning is performed according to the probability calculated as the relative particle weights of the two cells. For instance, if a particle moves into a cell whose particle weight is $80 \%$ of the weight for the original cell, then this particle will be cloned to 1.25 particles in the new cell, which is implemented as 1 or 2 particles with the help of a random number generator. The cloned particles share the same properties and, thus, affect the collision rate. The possible overall effects of this particle weighting strategy on the simulations are, therefore, investigated. First, several sets of particle weights are tested. One set is proportional to the cell volume, another is proportional to the square root of the cell volume, and a third set allows the simulation to have roughly 20 particles simulated in each cell. Second, a similar flow having zero flow velocity is simulated using the same particle weighting strategy. It is found that the main effect using the weight strategy is that the statistical scatter is increased due to the destroying and cloning. Another possible effect is that small numerical errors may be introduced. However, for the present investigation, the weight difference is not very large for low-altitude simulations, and the collision rate effect due to particle cloning is not important for high Knudsen number flows experienced at high altitudes. The resulting numerical errors are negligible for the present engineering analysis accounting for the statistical scatter and the geometry simplifications made. Therefore, the particle weighting strategy is considered acceptable for the present investigation.

\section{Flow Conditions}

Simulations are performed for the TOMEX flows at altitudes ranging from 85 to $143.27 \mathrm{~km}$ (apogee). The present DSMC simulations approximate the TOMEX flows as pseudosteady-state flows because the convective response of the flow is much faster than the variation of the flow conditions with altitude. The neutral temperature and densities of the atmosphere are estimated using the MSIS model $^{16}$ at 0957 UMT on 26 October 2000. Details of the flow conditions are listed in Table 1, in which $H$ is the altitude; $U$ and $W$ are the velocity components of the atmosphere relative to the payload in the $x$ and $z$ direction (in which the velocity component in $y$ direction is set to zero); whereas $n_{\mathrm{O}}, n_{\mathrm{N}_{2}}$, and $n_{\mathrm{O}_{2}}$ are the number densities of the atmosphere of atomic oxygen, molecular nitrogen, and molecular oxygen, respectively. (Minor constituents are not included in this study.) The Knudsen number is computed as the ratio of the mean free path of the freestream gas molecules to the diameter of the payload.

It is clear that the freestream velocity component normal to the payload axis was almost constant at roughly $200 \mathrm{~m} / \mathrm{s}$, whereas the velocity component parallel to the payload axis was decreased as the altitude increased. The temperature, however, decreased in the mesosphere and increased in the thermosphere with increasing altitude. The total number density of the atmosphere, on the other hand, dropped rapidly when the altitude increased. These conditions reveal that the TOMEX payload flew from supersonic, nearcontinuum flow at low altitude to subsonic, free-molecular flow at high altitude, which indicates the complexity of the TOMEX flows.

\section{E. Numerical Error}

In the present investigation, all of the simulations employ at least 15 particles in each cell, and the time step is less than the mean collision time of the atmosphere molecules. The total number of simulated particles is in the order of 10 million. A typical simulation runs for 20,000 time steps after the simulation reaches a steady state that generally takes less than 50,000 time steps. Each simulation requires approximately one day running on a 20-processor Linux cluster.

The simulation results are obtained by sampling the simulated particles, which means that the DSMC results exhibit statistical scatter that is sample-size dependent. The magnitude of the scatter, however, can only be estimated because the samples are statistically dependent and particle destroying and cloning may introduce additional scatter. Based on the simulations for a zero velocity flow and a general flow running many more time steps, the statistical error for the results presented in the next section is estimated to be less than $10 \%$ in general, which is considered acceptable given the simplifications made for the geometry modeling.

Table 1 Detailed flow conditions for simulations

\begin{tabular}{lcrcccccc}
\hline \hline$H, \mathrm{~km}$ & $U, \mathrm{~m} / \mathrm{s}$ & $W, \mathrm{~m} / \mathrm{s}$ & $T, \mathrm{~K}$ & $n_{\mathrm{O}}, 1 / \mathrm{m}^{3}$ & $n_{\mathrm{N}_{2}}, 1 / \mathrm{m}^{3}$ & $n_{\mathrm{O}_{2}}, 1 / \mathrm{m}^{3}$ & $M$ & $K n$ \\
\hline 85 & 205.72 & 1050.75 & 197.4 & $5.704 \times 10^{16}$ & $1.251 \times 10^{20}$ & $3.293 \times 10^{19}$ & 3.79 & 0.018 \\
90 & 205.12 & 1001.60 & 188.8 & $2.463 \times 10^{17}$ & $5.546 \times 10^{19}$ & $1.432 \times 10^{19}$ & 3.70 & 0.040 \\
95 & 204.75 & 953.29 & 181.9 & $4.607 \times 10^{17}$ & $2.358 \times 10^{19}$ & $5.870 \times 10^{18}$ & 3.58 & 0.093 \\
100 & 204.37 & 902.05 & 183.2 & $4.819 \times 10^{17}$ & $9.467 \times 10^{18}$ & $2.201 \times 10^{18}$ & 3.35 & 0.228 \\
105 & 204.09 & 848.63 & 200.1 & $3.610 \times 10^{17}$ & $3.673 \times 10^{18}$ & $7.627 \times 10^{17}$ & 2.99 & 0.592 \\
110 & 203.68 & 790.99 & 236.3 & $2.347 \times 10^{17}$ & $1.470 \times 10^{18}$ & $2.614 \times 10^{17}$ & 2.54 & 1.511 \\
115 & 203.35 & 728.79 & 297.3 & $1.456 \times 10^{17}$ & $6.345 \times 10^{17}$ & $9.477 \times 10^{16}$ & 2.06 & 3.612 \\
120 & 202.98 & 660.96 & 382.8 & $9.190 \times 10^{16}$ & $3.065 \times 10^{17}$ & $3.885 \times 10^{16}$ & 1.63 & 7.737 \\
125 & 202.71 & 585.25 & 460.1 & $6.405 \times 10^{16}$ & $1.749 \times 10^{17}$ & $1.938 \times 10^{16}$ & 1.32 & 13.76 \\
130 & 202.37 & 498.51 & 523.4 & $4.787 \times 10^{16}$ & $1.112 \times 10^{17}$ & $1.114 \times 10^{16}$ & 1.06 & 21.63 \\
135 & 201.97 & 393.48 & 579.1 & $3.722 \times 10^{16}$ & $7.539 \times 10^{16}$ & $7.009 \times 10^{15}$ & 0.82 & 31.63 \\
140 & 201.69 & 247.11 & 628.0 & $2.980 \times 10^{16}$ & $5.348 \times 10^{16}$ & $4.701 \times 10^{15}$ & 0.56 & 43.95 \\
143.27 & 201.50 & 1.87 & 656.7 & $2.612 \times 10^{16}$ & $4.357 \times 10^{16}$ & $3.717 \times 10^{15}$ & 0.35 & 53.32 \\
\hline \hline
\end{tabular}




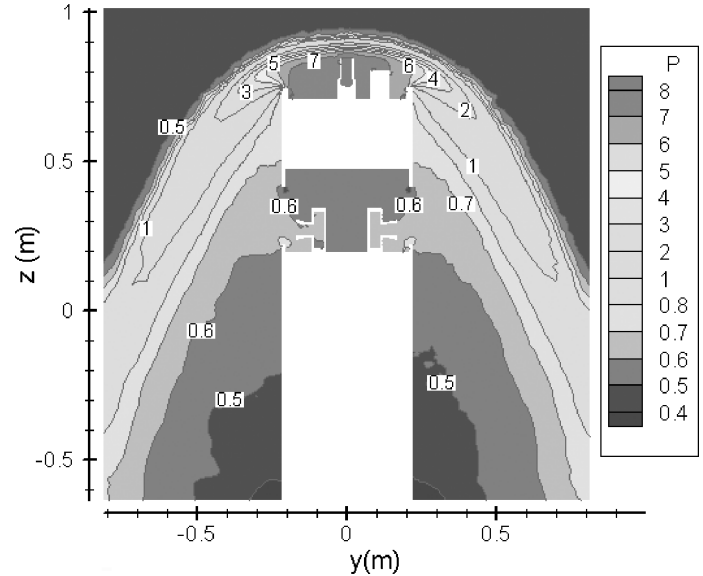

a) Pressure (pascal) contours in $x=0$ plane

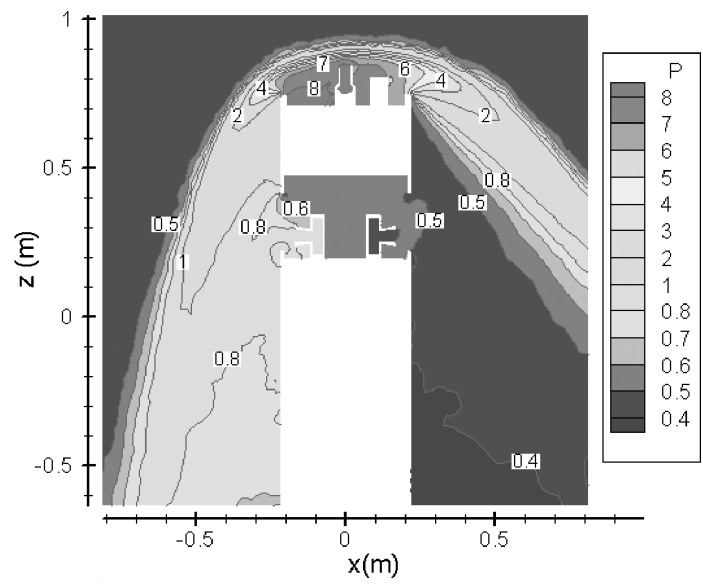

b) Pressure (pascal) contours in $y=0$ plane

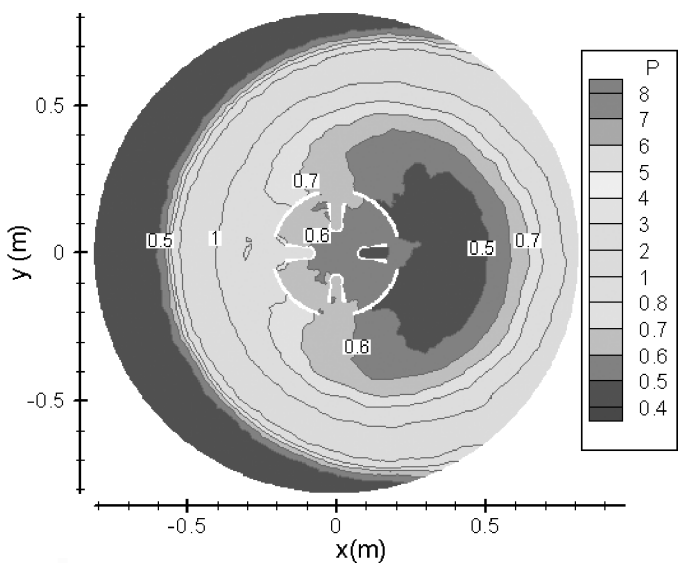

c) Pressure (pascal) contours in $z=0.275$ plane

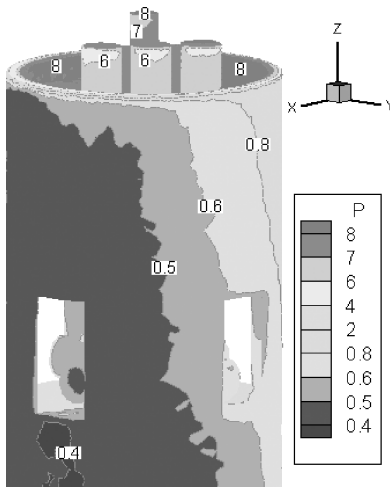

d) Surface pressure (pascal) contours

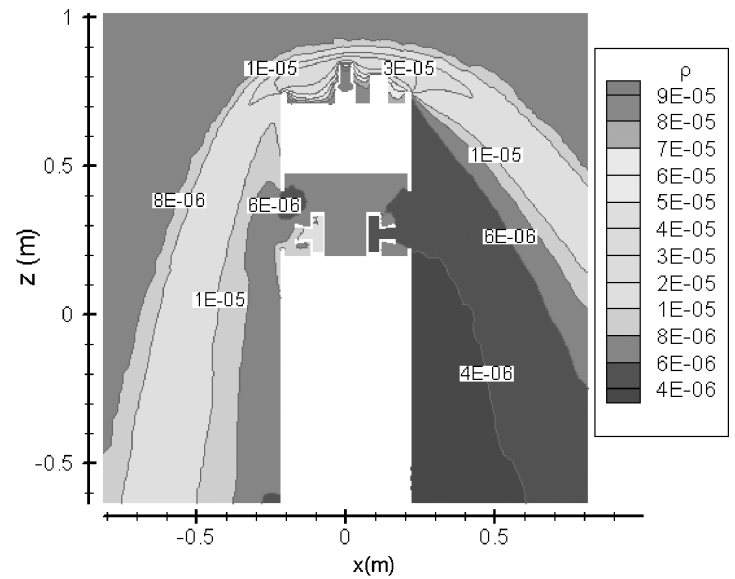

e) Density (kilograms per cubic meter) contours in $y=0$ plane

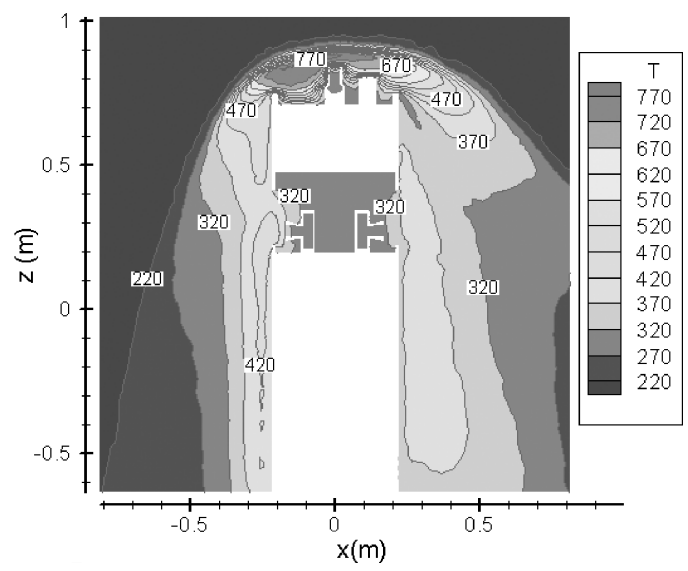

f) Temperature (Kelvin) contours in $y=0$ plane

Fig. 7 Flow patterns at $85 \mathrm{~km}$ predicted by DSMC simulation.

\section{Results and Discussion}

The DSMC method is applied to simulate the TOMEX flows at 13 different altitudes, and the simulation results show that the TOMEX flight generated complicated flow structures. To illustrate the flow pattern, the flow at $85 \mathrm{~km}$ is discussed as an example, and the pressure flow fields at several altitudes are analyzed. The simulation results are then compared with theoretical results when applicable. Comparison is also performed between the simulation results and measurement data.

\section{A. Flow Patterns}

The TOMEX flight was expected to generate complicated flow structures during the experiment, and this is predicted by the DSMC simulations. Figure 7 illustrates flow patterns for the case when the altitude is $85 \mathrm{~km}$, in which the Mach number of the freestream relative to the payload is roughly 3.8 . It is clear that a strong bow shock wave is generated (Figs. 7a-7c) about $0.1 \mathrm{~m}$ ahead of the payload. The bow shock wave is nearly symmetric in the $x=0$ plane, although the payload is unevenly distributed in the front, whereas it is nonsymmetric in the $y=0$ plane because of the 11-deg angle of attack. The pressure behind the shock wave is increased due to the shock compression. The increase reaches a maximum on the top of the payload and decreases downward along the payload surface. The 11-deg angle of attack of the flow also results in unevenly distributed pressure on the payload surface: The side of the payload facing toward the flow has a larger pressure, and the opposite side has a lower pressure, than that in a zero angle of attack case. Relatively 


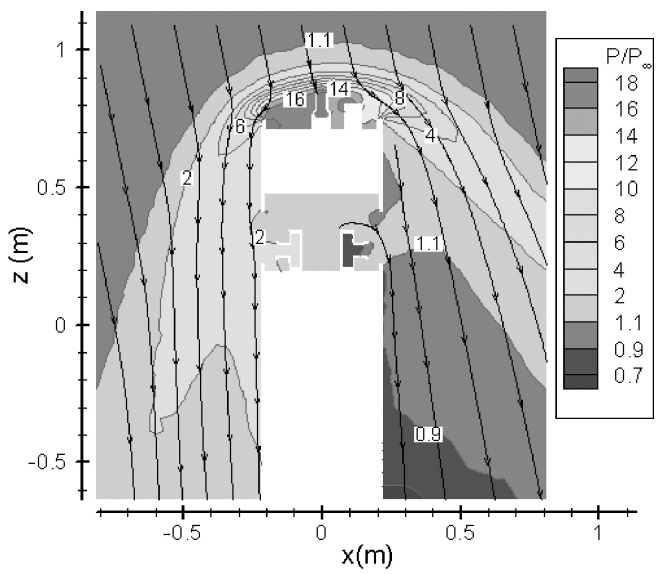

a) $90 \mathrm{~km}$

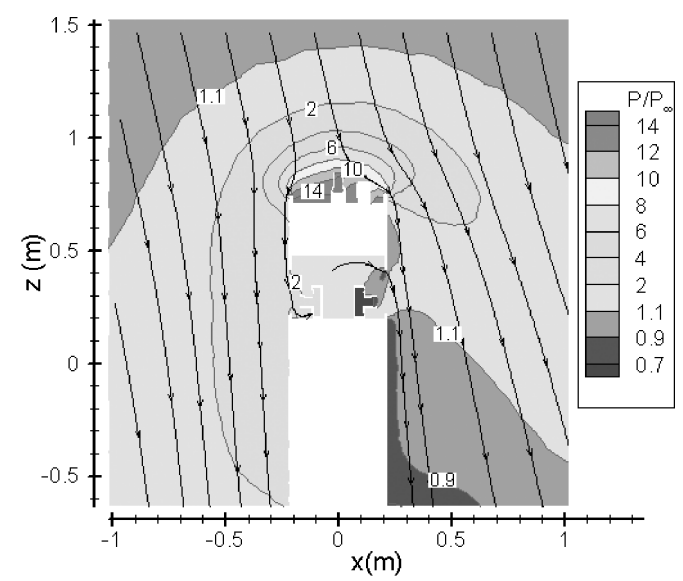

b) $100 \mathrm{~km}$

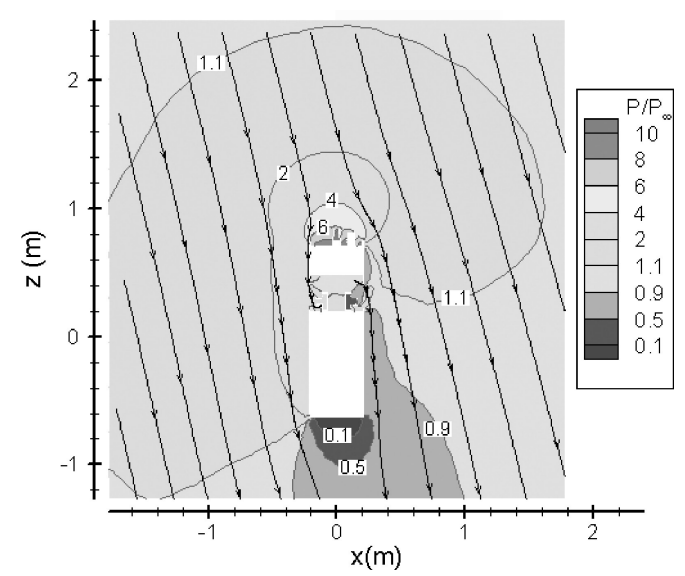

c) $110 \mathrm{~km}$

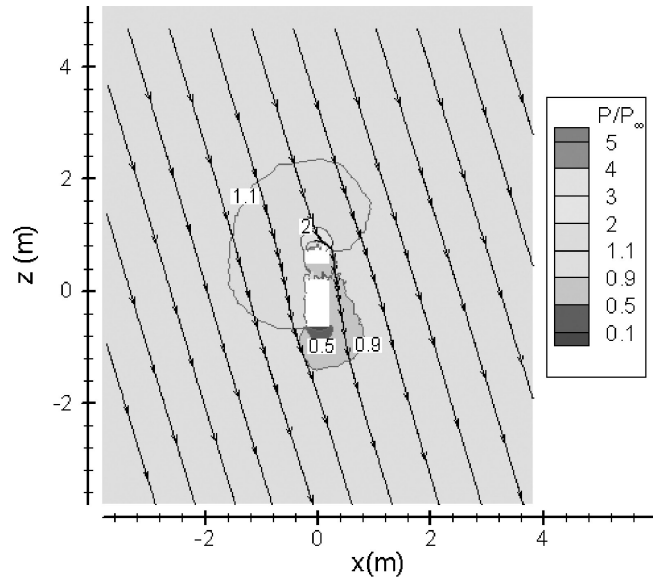

d) $120 \mathrm{~km}$

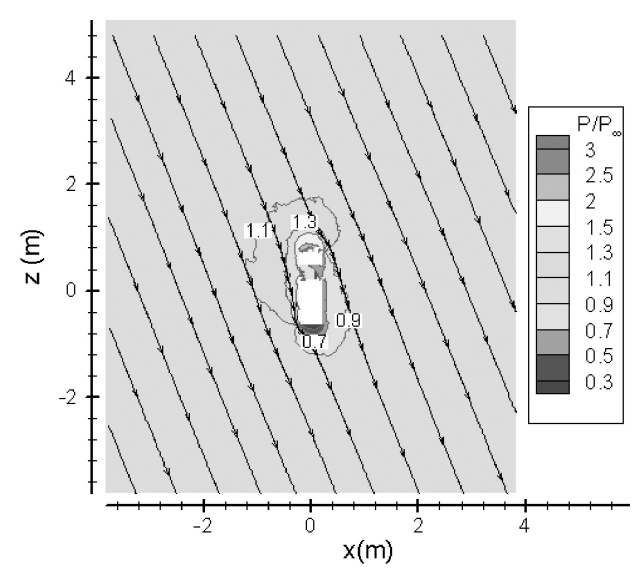

e) $130 \mathrm{~km}$

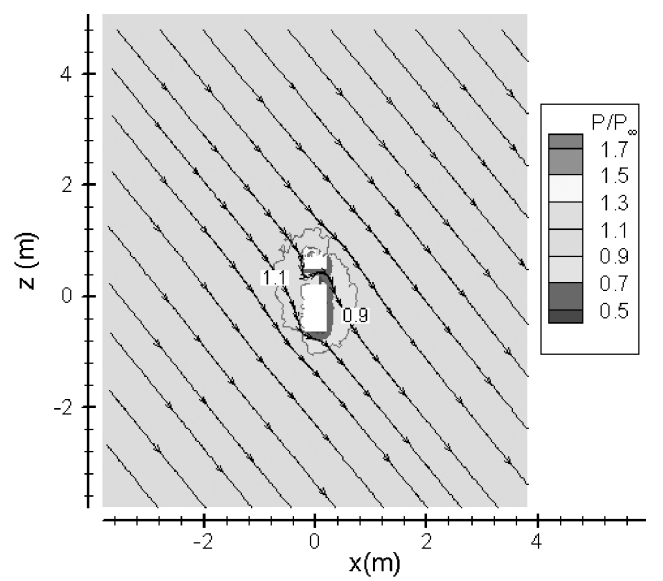

f) $140 \mathrm{~km}$

Fig. 8 Pressure contours in $y=0$ plane predicted by DSMC simulations.

low pressure is predicted inside the payload, whereas the pressure in the side channels of the ionization gauge depends on whether the opening faces the flow. Specifically, the channel located in the negative $x$ direction has a large pressure, and the one in the positive $x$ direction has a smaller pressure, whereas the pressures in the two channels located in the $x=0$ plane agree within numerical error. An overall pressure distribution is in Fig. 7d, which shows the pressure contours on the payload surface. Figures $7 \mathrm{e}$ and $7 \mathrm{f}$ show the density and temperature contours in the $y=0$ plane, respectively. The density shown is more similar to the pressure shown as compared with the temperature shown, mainly because the temperature on the surface is kept at $300 \mathrm{~K}$. However, a large temperature jump is found around the surface of the payload although the freestream Knudsen number is only 0.02 .
The flow patterns vary with altitude due to the changing flow conditions. First, the mean free path of the atmosphere is increased by three orders of magnitude from 85 to $143 \mathrm{~km}$, which changes the flow from the near-continuum regime to the free-molecular regime. The thickness of the bow shock wave, if it exists, will also increase because the thickness of a normal shock wave is roughly several mean free paths, which means that a shock wave in a rarefied condition can extend over a large distance. Also, the Mach number of the freestream is decreased from supersonic to subsonic.

The flow structures at several altitudes are illustrated in Fig. 8. Figures $8 \mathrm{a}-8 \mathrm{f}$ show the pressure contours normalized by the freestream pressure and representative streamlines in the $y=0$ plane where the velocity in the $y$ direction is very small. As shown, a shocklike structure, for example, pressure jump and total pressure 
drop, is still observed for the flow at $90 \mathrm{~km}$ (Fig. 8a), whereas a shock wave is hardly recognizable for the flow at $100 \mathrm{~km}$ (Fig. 8b) because the thickness of the shock wave becomes relatively large. Furthermore, the pressure plot for the flow at $110 \mathrm{~km}$ resembles that for the subsonic flow at $140 \mathrm{~km}$, although the freestream Mach number at $110 \mathrm{~km}$ is roughly 2.5 . Besides the differences in flow structures, it is also found that the pressure variation decreases as the altitude increases, and the variation is limited to a relatively small region at higher altitude. These results may indicate that the decrease of the pressure variation is mainly due to the decreasing freestream Mach number, because a rarefied flow is more compressible than a continuum flow.

\section{B. Pressure in Ionization Gauges}

The ionization gauge measures the neutral density of the gas in the vacuum tee, whereas the pressure is usually used to compare with data obtained using other techniques. Therefore, the pressure in the vacuum tee is analyzed, and comparisons are made among theoretical prediction, simulation results, and measurement data.

Inviscid compressible flow theory predicts the total pressure using the Mach number of the freestream ${ }^{20}$ :

$$
p_{0} / p_{\infty}=\left\{1+[(\gamma-1) / 2] M_{\infty}^{2}\right\}^{\gamma /(\gamma-1)}
$$

where $p_{0}$ is the total or stagnation pressure, $p_{\infty}$ is the freestream pressure, and $M_{\infty}$ is the freestream Mach number. For subsonic flows, $p_{0}$ can be regarded as the pressure in the vacuum tee of the top channel because the vacuum tee acts as a stagnation region. For supersonic flows, however, the total pressure drops across the shock wave.The pressure in the vacuum tee, or the total pressure behind the shock wave $p_{02}$, is approximated following the normal shock wave relations, as follows ${ }^{20}$ :

$$
\frac{p_{02}}{p_{0}}=\left[\frac{2 \gamma M_{\infty}^{2}-(\gamma-1)}{\gamma+1}\right]^{-1 /(\gamma-1)}\left[\frac{(\gamma+1) M_{\infty}^{2}}{2+(\gamma-1) M_{\infty}^{2}}\right]^{\gamma /(\gamma-1)}
$$

For pressures in the side channels, the inviscid theory is invalid because there are viscous interactions between the flow and the payload surface, which generally reduce the total pressure.

The pressure in the vacuum tee can also be approximately predicted by free-molecular theory because TOMEX flows at high altitude are in the free-molecular flow regime. The expression based on free-molecular theory is as follows ${ }^{21}$ :

$$
\begin{gathered}
p_{f}=p_{\infty}\left\{g\left(s_{3}\right) e^{-s_{3}^{2}}+\left[\frac{1}{2}+\sqrt{\pi} s_{3} g\left(s_{3}\right)\right]\left[1+\operatorname{erf}\left(s_{3}\right)\right]\right\} \\
g\left(s_{3}\right)=\left(s_{3} / \sqrt{\pi}\right)+\frac{1}{2} \sqrt{T_{r} / T_{\infty}}, \quad s_{3}=u_{3} \sqrt{m / 2 k T_{\infty}}
\end{gathered}
$$

where $p_{f}$ is the pressure in the vacuum tee, $T_{\infty}$ is the freestream temperature, $T_{r}$ is the reflected temperature (taken as the wall temperature), and $u_{3}$ is the velocity component normal to the door of the channel.

The pressure in the top channel of the ionization gauge is shown in Fig. 9, where the pressure is normalized by the freestream pressure. Figure 9 shows that the pressure predicted by the DSMC simulation is very close to that obtained using compressible flow theory when the altitude is below $105 \mathrm{~km}$, and it is a little smaller than that predicted by the free-molecular theory when the altitude exceeds $125 \mathrm{~km}$. Note that all three sets of results appear to converge at the highest altitudes. This is mainly due to the flow velocity decreasing below the sound speed toward zero and should not be taken to indicate that the continuum theory applies, in general, to highly rarefied flow.

The normalized pressure in the side channels of the ionization gauge is shown in Fig. 10. Here, the DSMC pressure approaches the free-molecular value when the altitude increases. The difference between the DSMC and free-molecular data at high altitude may be due to the effects of the presence of the payload surface and the structure of the ionization gauge, because the free-molecular expression [Eq. (3)] is obtained based on a simple flat-plate geometry. However, it seems that the simple free-molecular expression

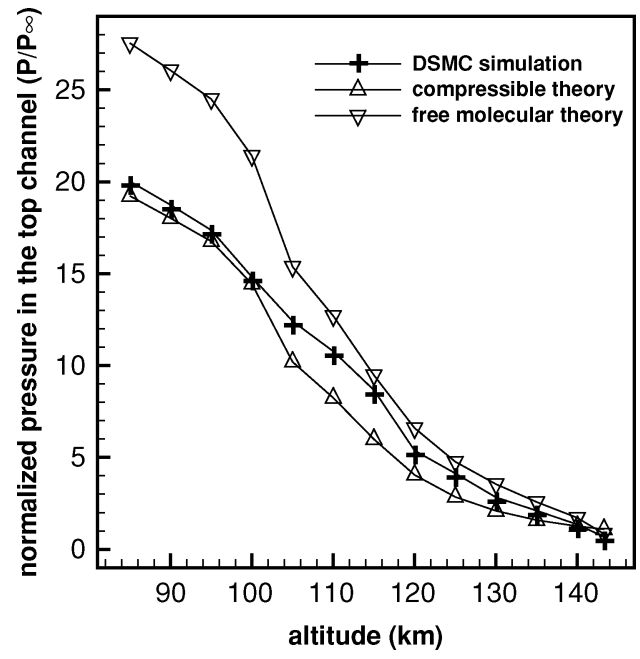

Fig. 9 Normalized pressure in top channel.

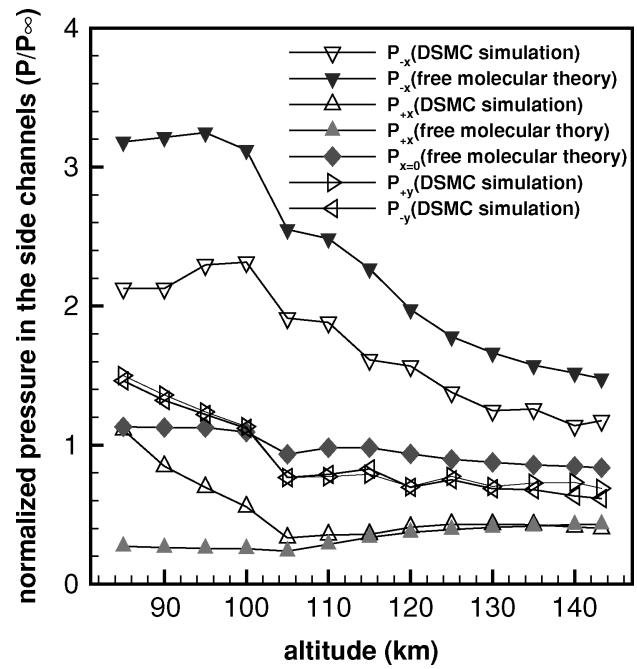

Fig. 10 Normalized pressure in side channels.

predicts very good results for the channel opposing the flow $P_{+x}$ when the altitude exceeds $120 \mathrm{~km}$, whereas the smaller pressure calculated using that expression at low altitude indicates that the free-molecular theory is invalid for low-altitude flow. In general, it can be concluded that the DSMC simulation results are very good because the results agree well with theoretical data when applicable.

\section{Pressure Comparison Between Simulation Results} and Measurement Data

The computed pressures in the channels of the ionization gauge are compared with the measurement data. During the TOMEX experiment, the payload had a spin rate of almost exactly $1 \mathrm{~Hz}$, which means that each of the side channels recorded the pressure that had a pseudofrequency of $1 \mathrm{~Hz}$.

The pressure data in the channels of the ionization gauge obtained from the DSMC simulations and the measurement are shown in Fig. 11. All of the measurement data have been calibrated and adjusted to account for effects such as gas venting from the interior of the payload. The simulation offers good agreement with the measurement data in the top channel, except at the lowest altitude. Possible reasons for the discrepancy are given in the next paragraph. The measured side data are taken from one of the side channels of the ionization gauge. The measured pressures in all side channels are very similar (differing mainly in phase), and their average is in Fig. 11. It is found that the DSMC simulation predicts a maximum pressure in the channel facing the flow and a minimum pressure in the channel opposing the flow, and these pressures bound the measurement data very well. 


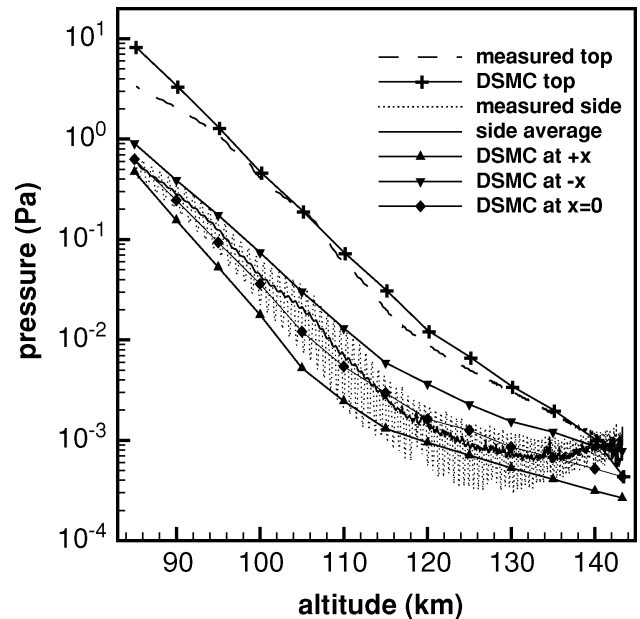

Fig. 11 Pressure comparison between DSMC results and measurements.

The slight difference between the simulation results and the measurement data is not surprising, accounting for possible differences of the flow conditions and payload configuration. For instance, the physical configuration of the payload is much more complicated than that modeled. No attempt has been made to model small internal features such as auxiliary parts and electrical cables. For example, the middle deck plate and the vacuum doors of the channels of the ionization gauge are removed in the simulation. Most important, the atmosphere conditions used in the simulation are based on the MSIS model, which may account for some of the difference between measurement and simulation data. The data obtained from the lidar measurement indicate that the temperature of the atmosphere below $92 \mathrm{~km}$ was a little warmer than that given by the MSIS model. One outcome is that the simulation pressure in the top channel will decrease by roughly $16 \%(85-\mathrm{km}$ case) if the pressure of the atmosphere is kept the same while the temperature measured by lidar is used in the simulation. The decrease of the channel pressure occurs in this case because the freestream Mach number is decreased due to the warmer atmosphere. When all of these factors, are accounted for, the DSMC simulations are very successful for studying the TOMEX flows and show flow details that are not revealed by the measurement; on the other hand, the ionization gauge can successfully measure the atmosphere conditions.

\section{Conclusions}

The DSMC method was used to analyze the three-dimensional flows ranging from near-continuum to free-molecular flow regimes that were experienced during the TOMEX. Both the external and internal flows were simulated using several complex tetrahedral meshes, where detailed structures of the payload were modeled.

The DSMC simulations employed a particle-weighting technique to limit the total number of simulated particles and a near-partner selection strategy to determine the collision particle pairs. It was found that the flows were fully three dimensional due to the nonsymmetric payload geometry and nonzero angle of attack of the flows. A bow shock wave was generated in front of the payload at low altitude that became diffusive when the altitude was increased, even when the Mach number of the freestream was larger than 2.5. It was also found that the pressure in the channels of the ionization gauge decreased monotonically with increasing altitude, where the pressure variation was also decreased.

The pressures at measurement locations were estimated using compressible flow theory and free-molecular theory and indicated that the DSMC results were reasonable. Good agreement was also obtained between the DSMC results and measurement data. It was concluded that the DSMC simulations predicted the TOMEX flows very well, which indicates that the DSMC method, an important tool in the analysis and interpretation, should be used as part of the design and analysis process for future flight experiments.

\section{Acknowledgments}

This work is funded in part by NASA through Grants NAG55235 and NAG5-5412. The authors are also grateful for computational resources provided on the Maui High Performance Computing Center and on the high-performance clusters in the Department of Aerospace Engineering and Mechanics at the University of Minnesota.

\section{References}

${ }^{1}$ Hecht, J. H., Liu, A. Z., Bishop, R. L., Clemmons, J. H., Gardner, C. S., Larsen, M. F., Roble, R. G., Swenson, G. R., and Walterscheid, R. L., "An Overview of Observations of Unstable Layers During the Turbulent Oxygen Mixing Experiment (TOMEX)," Journal of Geophysical Research, Vol. 109, No. D2, 2004, D02S01.

${ }^{2}$ Bird, G. A., Molecular Gas Dynamics and the Direct Simulation of Gas Flows, Oxford Univ. Press, New York, 1994.

${ }^{3}$ Rault, D. F. G., "Aerodynamics of the Shuttle Orbiter at High Altitudes," Journal of Spacecraft and Rockets, Vol. 31, No. 6, 1994, pp. 944-952.

${ }^{4}$ Hass, B. L., and Schmitt, D. A., "Simulated Rarefied Aerodynamics of the Magellan Spacecraft During Aerobraking," Journal of Spacecraft and Rockets, Vol. 31, No. 6, 1994, pp. 980-985.

${ }^{5}$ Wilmoth, R. G., Mitcheltree, R. A., and Moss, J. N., "Low-Density Aerodynamics of the Stardust Sample Return Capsule," Journal of Spacecraft and Rockets, Vol. 36, No. 3, 1999, pp. 436-441.

${ }^{6}$ Ivanov, M. S., Markelov, G. N., Gimelshein, S. F., Mishina, L. V., Krylov, A. N., and Grechko, N. V., "High-Altitude Capsule Aerodynamics with Real Gas Effects," Journal of Spacecraft and Rockets, Vol. 35, No. 1, 1998, pp. 16-22.

${ }^{7}$ Moss, J. N., Blanchard, R. C., Wilmoth, R. G., and Braun, R. D., "Mars Pathfinder Rarefied Aerodynamics: Computations and Measurements," Journal of Spacecraft and Rockets, Vol. 36, No. 3, 1999, pp. 330-339.

${ }^{8}$ Boyles, K. A., LeBeau, G. J., and Gallis, M. A., "DSMC Simulation in Support of the Columbia Shuttle Orbiter Accident Investigation," AIAA Paper 2004-2282, June 2004.

${ }^{9}$ Gallis, M. A., Hermina, W. L., Johnson, M. A., and Chapel, J. D., "Aerothermal Analysis for the Mars Reconnaissance Orbiter," AIAA Paper 2004-2684, June 2004.

${ }^{10}$ Gatsonis, N. A., Maynard, E. P., and Erlandson, R. E., "Monte Carlo Modeling and Analysis of Pressure Sensor Measurements During Suborbital Flight,"Journal of Spacecraft and Rockets, Vol. 34, No. 1, 1997, pp. 83-91.

${ }^{11}$ Liu, A. Z., Roble, R. G., Hecht, J. H., Larsen, M. F., and Gardner, C. S., "Unstable Layers in the Mesopause Region Observed with Na Lidar During the Turbulent Oxygen Mixing Experiment (TOMEX) Campaign," Journal of Geophysical Research, Vol. 109, No. D2, 2004, D02S02.

${ }^{12}$ Bishop, R. L., Larsen, M. F., Hecht, J. H., Liu, A. Z., and Gardner, C. S., "TOMEX: Mesospheric and Lower Thermospheric Diffusivities and Instability Layers," Journal of Geophysical Research, Vol. 109, No. D2, 2004, D02S03.

${ }^{13}$ Hecht, J. H., Liu, A. Z., Walterscheid, R. L., Roble, R. G., Larsen, M. F., and Clemmons, J. H., "Airglow Emissions and Oxygen Mixing Ratios from the Photometer Experiment on the Turbulent Oxygen Mixing Experiment (TOMEX)," Journal of Geophysical Research, Vol. 109, No. D2, 2004, D02S05.

${ }^{14}$ Larsen, M. F., Liu, A. Z., Bishop, R. L., and Hecht, J. H., "TOMEX: A Comparison of Lidar and Sounding Rocket Chemical Tracer Wind Measurements," Geophysical Research Letters, Vol. 30, No. 7, 2003, pp. 1375-1378.

${ }^{15}$ Sicinski, H. S., Spencer, N. W., and Dow, W. G., "Rocket Measurements of Upper Atmosphere Ambient Temperature and Pressure in the 30-Kilometer to 75-Kilometer Region,' Journal of Applied Physics, Vol. 25, No. 2, 1954, pp. 161-168.

${ }^{16}$ Hedin, A. E., "Extension of the MSIS Thermospheric Model into the Middle and Lower Atmosphere," Journal of Geophysical Research, Vol. 96, No. A2, 1991, pp. 1159-1172.

${ }^{17}$ Dietrich, S., and Boyd, I. D., "Scalar and Parallel Optimized Implementation of the Direct Simulation Monte Carlo Method," Journal of Computational Physics, Vol. 126, No. 2, 1996, pp. 328-342.

${ }^{18}$ Karypis, G., and Kumar, V., "METIS, A Software Package for Partitioning Unstructured Graphs, Partitioning Meshes, and Computing FillReducing Orderings of Sparse Matrices, version 4.0," Dept. of Computer Science and Engineering, Technical Rept., Univ. of Minnesota, Minneapolis, MN, 1998

${ }^{19}$ Hypermesh, Ver. 5.0, Altair Engineering, Inc., Troy, MI, 2003.

${ }^{20}$ Anderson, J. D., Modern Compressible Flow: with Historical Perspective, McGraw-Hill, New York, 1990, Chap. 3.

${ }^{21}$ Gombosi, T. I., Gaskinetic Theory, Cambridge Univ. Press, Cambridge, England, U.K., 1994, Chap. 7. 